XLVIth Zakopane School of Physics, International Symposium Breaking Frontiers, Zakopane, Poland, May 16-21, 2011

\title{
Initial Phases of Antarctic Ramalina terebrata Hook f. \& Taylor Thalli Rehydration Observed by Proton Relaxometry
}

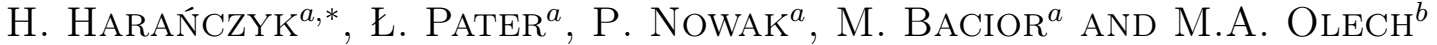 \\ ${ }^{a}$ Institute of Physics, Jagiellonian University, W.S. Reymonta 4, 30-059 Kraków, Poland \\ ${ }^{b}$ Institute of Botany, Jagiellonian University, M. Kopernika 27, 31-501 Kraków, Poland
}

\begin{abstract}
Hydration kinetics, sorption isotherm, and proton free induction decays are measured for Ramalina terebrata thalli rehydrated from gaseous phase. Very tightly, tightly, and loosely bound water fractions are distinguished. Sorption isotherm is sigmoidal in form with the mass of water saturating primary water binding sites equal to $\Delta m / m_{0}=0.046$. Proton free induction decays show the presence of immobilized water fraction $\left(T_{2 L_{1}}^{*} \approx 100 \mu \mathrm{s}\right)$ and mobile water pool $\left(T_{2 L_{2}}^{*} \approx 330 \mu \mathrm{s}\right)$. Sorption isotherm fitted to the NMR data shows the absence of water fraction "sealed" in pores of dry thallus.
\end{abstract}

PACS: $82.56 . \mathrm{Na}$

\section{Introduction}

Antarctic lichens are extremophilic organisms surviving extremely low temperatures [1-3] and deep dehydration of their thalli [4-7]. They may reversibly dehydrate below two-dimensional water percolation on the inner surfaces of thallus [8]. Antarctic lichen taxa may be photosynthetically active and productive even if tissue is frozen [9-12], and can hydrate from the gaseous phase to the hydration level sufficient to initiate photosynthesis [12]. As both drought and cold resistance may have similar molecular mechanism, the thallus dehydration is one of ways to resist deeply decreased temperature that lichens experience in their habitat.

To understand the molecular mechanism of the metabolic activity recovery during rehydration of thallus one needs to know a number and a distribution of water binding sites, sequence and kinetics of their saturation, and the formation of tightly and loosely bound water fractions at different steps of hydration process.

\section{Materials and methods}

Ramalina terebrata Hook f. \& Taylor is fruticose lichen with flattened branches, simple or branched. Both sides of thallus corticate have perforations and numerous elongated or rounded respiratory pores called pseudocyphellae $[13,14]$. It grows on coastal rocks, usually close to bird colonies, especially to penguin rockeries, in the maritime Antarctic [14].

Samples of the lichen Ramalina terebrata were collected in the vicinity of Arctowski Station, King George Island, maritime Antarctica, on 27th December 1987 year. Air-dry thalli were stored in herbarium at the room temperature. The hydration level of air-dry thalli

\footnotetext{
* corresponding author; e-mail: hubert.haranczyk@uj.edu.pl
}

was equal to $\Delta m / m_{0}=0.096$. As a measure of hydration level, the relative mass increase, $\Delta m / m_{0}$, expressed in units of dry mass, was taken.

Before the hydration courses the thallus was incubated for 11 days over silica gel $\left(p / p_{0}=0 \%\right)$, reaching the hydration level equal to $\Delta m / m_{0}=0.048 \pm 0.004$. The hydration time-courses were performed from the gaseous phase, at room temperature $\left(t=22^{\circ} \mathrm{C}\right)$ over the surface of: $\mathrm{H}_{3} \mathrm{PO}_{4}\left(p / p_{0}=9 \%\right)$, over the surface of supersaturated solutions of $\mathrm{CaCl}_{2}\left(p / p_{0}=32 \%\right), \mathrm{Na}_{2} \mathrm{Cr}_{2} \mathrm{O}_{7}$ $\left(p / p_{0}=52 \%\right), \mathrm{Na}_{2} \mathrm{~S}_{2} \mathrm{O}_{3}(76 \%), \mathrm{K}_{2} \mathrm{CrO}_{3}(88 \%), \mathrm{Na}_{2} \mathrm{SO}_{4}$ $(93 \%)$, and over the water surface $\left(p / p_{0}=100 \%\right)$. After completing of the hydration courses, the dry mass of the thallus was determined after heating at $70^{\circ} \mathrm{C}$ for $72 \mathrm{~h}$. Higher temperatures were not used as they may cause the decomposition of some organic constituents of thallus [15].

The NMR measurements were performed at room temperature $\left(t=22^{\circ} \mathrm{C}\right)$ using high power WNS HB-65 relaxometer (Waterloo NMR Spectrometers, St. Agatha, Ontario, Canada). The resonance frequency was $30 \mathrm{MHz}$ (at the field strength of $0.7 \mathrm{~T}$ ); the transmitter power was $400 \mathrm{~W}$; the pulse length $\pi / 2=1.4 \mu \mathrm{s}$. The power of the pulse was sufficient to observe the total proton signal (including protons from solid state of thallus). Free induction decays (FIDs) were acquired using Compuscope 2000 card of an IBM 80486 clone controlling the spectrometer and averaged over 2000 accumulations. Repetition time was $2.003 \mathrm{~s}$. The data obtained were analyzed using the FID analyzing procedure of a two-dimensional (in time domain) NMR signal-analyzing software CracSpin written at the Jagiellonian University, Cracow [16].

For hydration kinetics measurements the fluctuations of illumination in laboratory cycle: day/night occurred, whereas the NMR relaxation measurements were performed at night conditions, as no additional light supply was introduced to the probe of the spectrometer. 


\section{Results}

The hydration courses of the Ramalina terbrata thallus performed at the relative humidity between $p / p_{0}=9 \%$ and $52 \%$ were fitted well by a one-exponential function, whereas for higher humidity were fitted well by the two-exponential function (Fig. 1):

$$
\Delta m / m_{0}=C^{\mathrm{h}}-A_{1}^{\mathrm{h}} \exp \left(-t / t_{1}^{\mathrm{h}}\right)-A_{2}^{\mathrm{h}} \exp \left(-t / t_{2}^{\mathrm{h}}\right),
$$

where $A_{0}^{\mathrm{h}}=C^{\mathrm{h}}-A_{1}^{\mathrm{h}}-A_{2}^{\mathrm{h}}$ is the saturation level at incubation $\left(p / p_{0}=0 \%\right), A_{1}^{\mathrm{h}}$ and $A_{2}^{\mathrm{h}}$ are the saturation hydration levels for the fast and slow component, $t_{1}^{\mathrm{h}}$ and $t_{2}^{\mathrm{h}}$ are hydration times for fast and slow component, respectively.

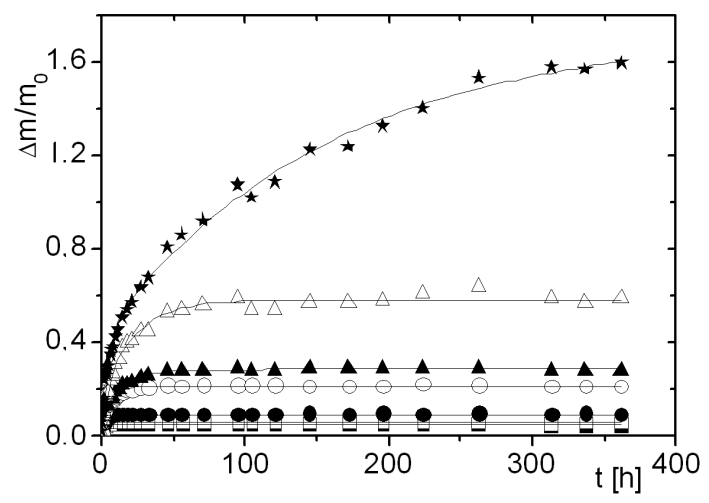

Fig. 1. The rehydration of the lichen Ramalina terebrata from gaseous phase at different values of relative humidity $p / p_{0}$ recorded as the relative mass increase expressed in units of dry mass, $\Delta m / m_{0}$. Target humidities: $p / p_{0}=9 \%$ - closed squares, $p / p_{0}=32 \%$ - open squares, $p / p_{0}=52 \%$ - closed spheres, $p / p_{0}=76 \%$ open spheres, $p / p_{0}=88 \%$ - closed triangles, $p / p_{0}=$ $93 \%$ - open triangles, $p / p_{0}=100 \%$ - closed starlets. The errors are within the plot symbols.

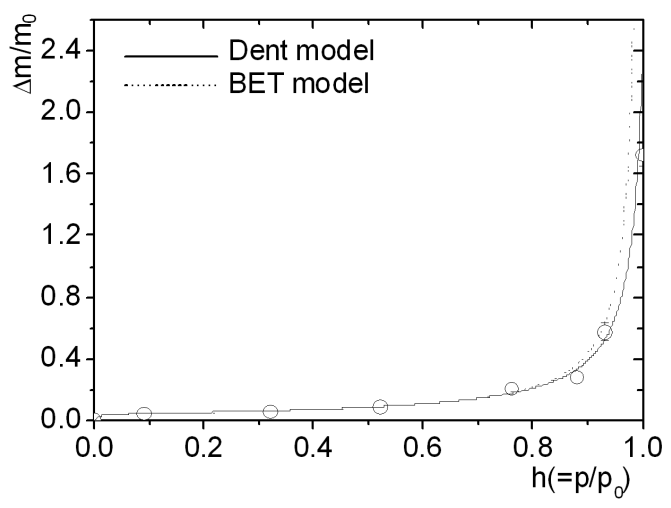

Fig. 2. The sorption isotherm for Ramalina terebrata. The values of $h\left(=p / p_{0}\right)$ represent the relative humidity and the values of relative mass increase, $\Delta m / m_{0}$, are defined as the $C^{\mathrm{h}}$ values are taken from Eq. (1).

The averaged over the all hydration courses value of $A_{0}^{\mathrm{h}}=0.007 \pm 0.001$, is the saturation level for very tightly bound water fraction level not removed by incubation over silica gel $\left(p / p_{0}=0 \%\right)$, and binding to the surfaces with a very short hydration time. The values of parameters are equal to: $A_{1}^{\mathrm{h}}=0.059 \pm 0.007, t_{1}^{\mathrm{h}}=(1.24 \pm 0.24) \mathrm{h}$, and $t_{2}^{\mathrm{h}}=(14.4 \pm 2.1) \mathrm{h}$. The saturation level of the $A_{2}^{\mathrm{h}}$ component increases gradually with increased humidity. The saturation hydration level, $C^{\mathrm{h}}$ at given relative humidity, $p / p_{0}$, was taken for sorption isotherm.

For the Ramalina terebrata thalli the sorption isotherm reveals a sigmoidal form (Fig. 2), fitted better by the Dent model [17] (Eq. (2)), rather than by the BrunauerEmmett-Teller (BET) model [18]. Both of these models distinguish: (i) "primary" water binding sites (directly to the adsorbent surface); and (ii) "secondary", usually weaker, water binding sites (to the primary bound water molecules, or to the next water layers):

$$
C^{\mathrm{h}}(h)=\frac{\Delta M}{m_{0}} \frac{b_{1} h}{(1-b h)\left(1+b_{1} h-b h\right)},
$$

where $h=p / p_{0}$ is relative humidity expressed as a ratio, $\Delta M / m_{0}$ is the mass of water saturating primary water binding sites and for $R$. terebrata it equals: $\Delta M / m_{0}=$ 0.046. In BET model the coverage of the $n$-th water layer expressed in units of the coverage of $(n-1)$-th: $S_{n} /\left.S_{n-1}\right|_{h=1}=1$, whereas the Dent model allows the decrease of the coverage for the subsequent water layers introducing an additional parameter $b=S_{n} /\left.S_{n-1}\right|_{h=1}$. The value of $b$ may vary from 1 down to 0 . For $R$. terebrata equal to 0.941 , where $S_{i}$ is the population of $i$-th water layer, and the contribution of empty primary binding sites at $h=1$ is expressed by the reciprocal of $b_{1}$ : $S_{0} /\left.N\right|_{h=1}=1 / b_{1}$, where $S_{0}$ is the number of water binding sites on the surface, $1 / b_{1}=0.6 \%$. For other investigated lichens, this value equals $1 / b_{1}=0.02 \%$ (Umbilicaria aprina) [19], $1 / b_{1}=0.76 \%$ (Usnea antarctica) [8].

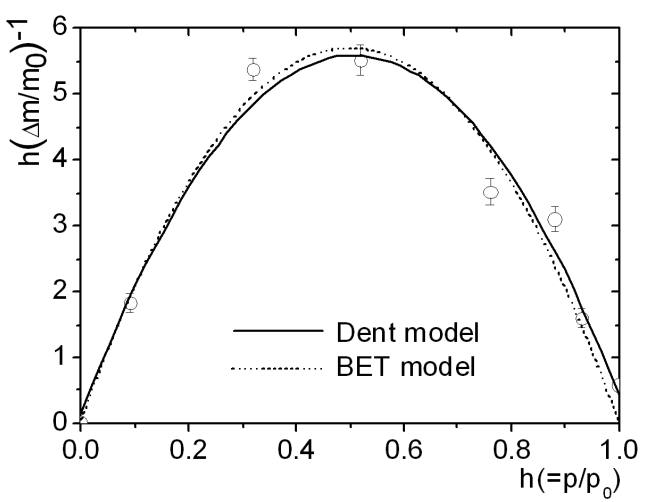

Fig. 3. Parabolic form of sorption isotherm for Ramalina terebrata thalli (open circles - experimental data, solid line - the Dent model, dotted line - the BET model).

BET model gives the similar results for $\Delta M / m_{0}=$ 0.044 . Fitted value of $1 / b_{1}$ is three times smaller than for the Dent model, and equals $1 / b_{1}=0.2 \%$. Usually sorption isotherms are presented in parabolic forms (see 
Fig. 3) which are easier to intuitive estimation of the applicability of both models

$$
\begin{aligned}
& \frac{h}{\Delta m / m_{0}}=A+B h-(A+B) h^{2}, \\
& \frac{h}{\Delta m / m_{0}}=A+B h-C h^{2} .
\end{aligned}
$$

For the Dent model the parameters $\Delta M / m_{0}, b, b_{1}$ are connected with $A, B, C$ by the formulae

$$
b=\frac{\sqrt{B^{2}+4 A C}-B}{2 A}, \quad b_{1}=\frac{B}{A}+2 b, \quad \frac{\Delta M}{m_{0}}=\frac{1}{A b_{1}} .
$$

The criterion for distinguishing between the BET and Dent model applicability is the value of sorption isotherm expressed in form of Eq. (3a) or $(3 \mathrm{~b})$ for $h=1$. If BET model applies well the function crosses $X$-axis at $h=1$. The difference from zero at $h=1$ is the measure of the Dent model applicability.

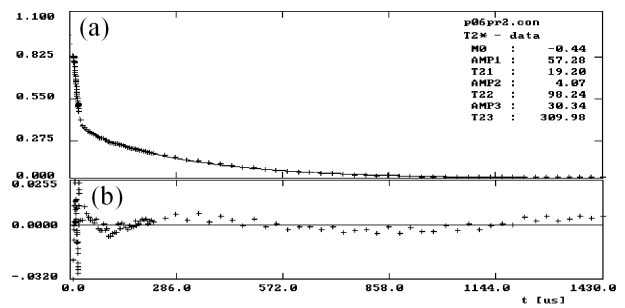

Fig. 4. (a) Proton free induction decay recorded for the thallus Ramalina terebrata. The relative mass increase was $\Delta m / m_{0}=16.5 \%$. $Y$-axis shows the normalized signal amplitude. The solid line represents a least squares fit of Eq. (5) to the data with the fitted parameters given in the figure legend $\left(T 21=T_{2 S}^{*}, T 22=T_{2 L_{1}}^{*}\right.$, $T 23=T_{2 L_{2}}^{*}, \mathrm{Amp} 1=S, \mathrm{Amp} 2=L_{1}$ and Amp3 $\left.=L_{2}\right)$. (b) The residual function calculated as the difference between the fitted and recorded values of the FID signal, which does not exceed $2.6 \%$ for any recorded point.

Figure 4 shows the proton free induction decay (FID) recorded at room temperature for a thallus of $R$. terebrata rehydrated to $\Delta m / m_{0}=16.5 \%$. The recorded proton FID function does not reveal the "beat" pattern characteristic for solid component fitted by the Abragam function $[20,21]$, so is well fitted by the superposition of the Gaussian component, with the amplitude $S$, coming from the solid matrix of thallus; and one with the amplitude equal to $L_{1}$; or two, $L_{1}$ and $L_{2}$, exponential components coming from tightly and loosely bound water fractions to the surfaces of thallus

$$
\begin{aligned}
& \operatorname{FID}(t)=S \exp \left(-\left(\frac{t}{T_{2 S}^{*}}\right)^{2}\right)+L_{1} \exp \left(-\frac{t}{T_{2 L_{1}}^{*}}\right) \\
& +L_{2} \exp \left(-\frac{t}{T_{S L_{2}}^{*}}\right),
\end{aligned}
$$

where $T_{2 S}^{*}$ is proton relaxation time of solid component taken at 1 /e-value of Gaussian solid signal, and
$T_{2 L_{1}}^{*}$ and $T_{2 L_{2}}^{*}$ are the relaxation times of proton liquid fractions $L_{1}$ and $L_{2}$, respectively. The $L_{1}$ signal component with the value $T_{2 L_{1}}^{*} \approx 100 \mu$ s is characteristic of tightly bound water fraction either lichen thalli or for many other biological systems [19, 22-30]. The $L_{2}$ signal with $T_{2 L_{2}}^{*} \approx 330 \mu \mathrm{s}$, shortened by $B_{0}$ inhomogeneities [31], comes from water loosely bound on thallus surfaces and/or from free water fraction (Fig. 5).

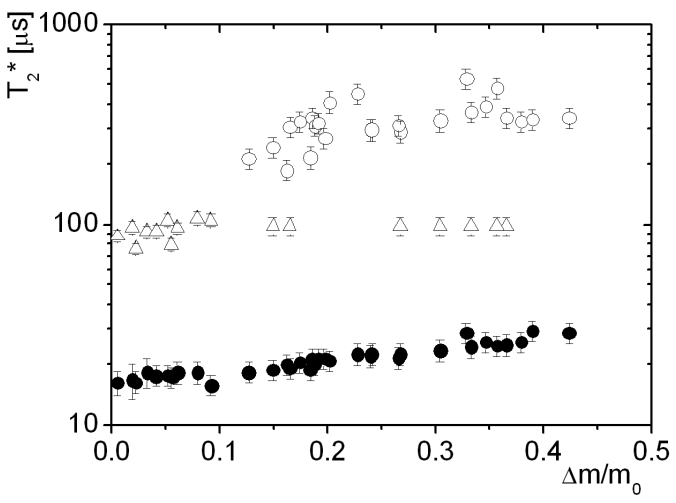

Fig. 5. The hydration dependence of proton FID relaxation times for Ramalina terebrata.

For $R$. terebrata thallus the relaxation time $T_{S}^{*} \approx 20 \mu \mathrm{s}$ for Gaussian component does not depend much on hydration level (Fig. 5), and is close to the value for other lichen thalli, and different solid tissues as dentine, dental enamel, the shells of molluscs, bark and bast $([32,19]$ and the references therein). This correspondence observed in a wide range of biological systems comes from general congeniality of proton chemical environments over angstrom dimensional scales [31, 33]. Thus it can be used as a unit for scaling of liquid components.

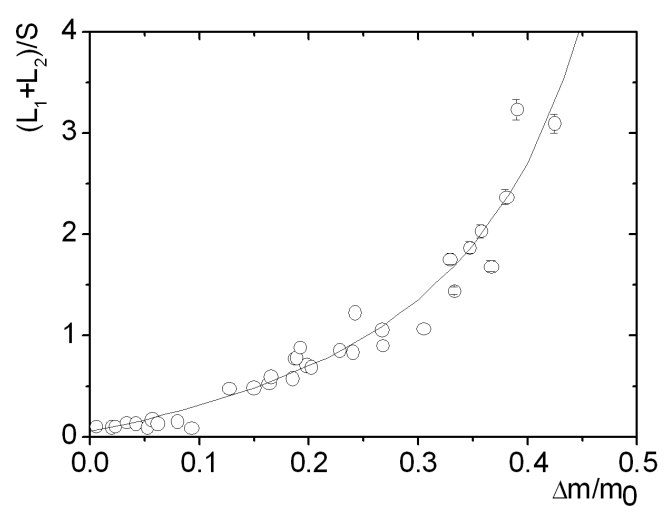

Fig. 6. The total liquid signal, $\left(L_{1}+L_{2}\right) / S$, expressed in units of solid signal hydration dependence for Ramalina terebrata.

The hydration dependence of total liquid signal, expressed in the units of solid, $L / S$, is well fitted by the rational function (Fig. 6) 


$$
\begin{aligned}
& \frac{L_{1}+L_{2}}{S}\left(\Delta m / m_{0}\right) \\
& =\frac{(-0.05 \pm 0.07)+(2.05 \pm 0.38) \Delta m / m_{0}}{1+(-1.70 \pm 0.10) \Delta m / m_{0}} .
\end{aligned}
$$

This type of function suggests the presence of solid water soluble fraction dissolving in water with increasing hydration of the sample [32], which in lichen thalli consists of sugars and/or polyols [34]. The hydration dependence of "second" exponential component, $L_{2} / S$, expressed in the units of solid, $S$, is presented in Fig. 7 and for $\Delta m / m_{0} \geq 0.15$ is fitted by the rational function

$$
\begin{aligned}
& \frac{L_{2}}{S}\left(\Delta m / m_{0}\right) \\
& \quad=\frac{(-0.21 \pm 0.29)+(0.73 \pm 1.67) \Delta m / m_{0}}{1+(-1.91 \pm 0.63) \Delta m / m_{0}} .
\end{aligned}
$$

The total liquid signal expressed in units of solid, $L / S$, may be used to construct the NMR-isotherm, with the shape described by the same sorption parameters $\left(\Delta M / m_{0}, b\right.$, and $\left.b_{1}\right)$ as the sorption isotherm obtained from the gravimetry (Fig. 8). In such a case the NMR signal intensities are fitted by the function

$$
\frac{L_{1}+L_{2}}{S}(h)=A+k \frac{\Delta M}{m_{0}} \frac{b_{1} h}{(1-b h)\left(1+b_{1} h-b h\right)},
$$

where $k=7.14 \pm 0.28$ and $A=0$.

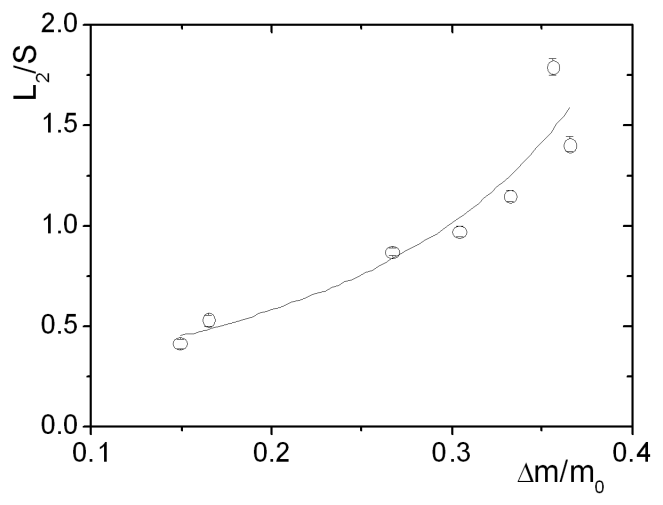

Fig. 7. The loosely bound water signal, $L_{2} / S$, in units of solid hydration dependence for Ramalina terebrata thallus.

If the effect of paramagnetic ions present in the system differs with the proximity to the membrane surface, the sorption isotherm should be modeled with two proportionality coefficients, $k_{1}$ for tightly bound water fraction, and $k_{2}$ for loosely bound water fraction (Eq. (9)):

$$
\begin{aligned}
& \frac{L_{1}+L_{2}}{S}(h)=A+\frac{\Delta M}{m_{0}} \frac{b_{1} h}{1+\left(b_{1}-b\right) h} \\
& \quad \times\left(k_{1}+k_{2} \frac{b h}{1-b h}\right) .
\end{aligned}
$$

The sorption isotherm is well fitted to the NMR data (Eq. (9)), for $k_{1}=2.5, k_{2}=4$ and $A=0$.

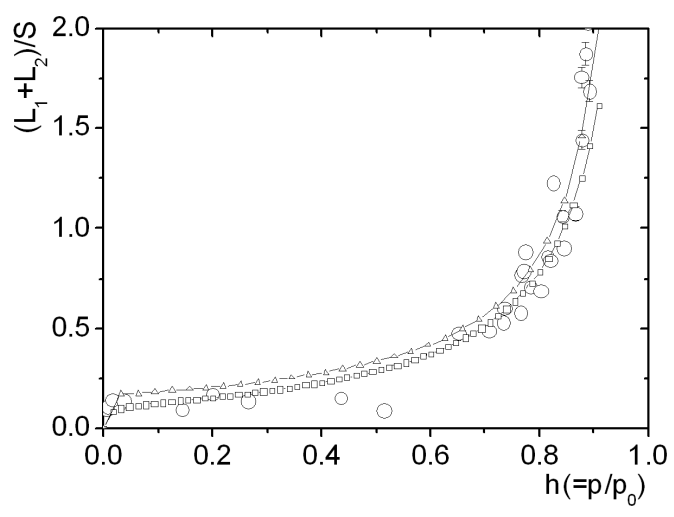

Fig. 8. Sorption isotherm fitted to total FID proton liquid component signal for water hydrating thallus of Ramalina terebrata. Open triangles - the Dent model with one $k$ coefficient; open squares - the Dent model with two $k$ coefficients.

Parameter $A$ describes the contribution of liquid signal in dry $R$. terebrata. For some dry biological systems (e.g. freeze-dried photosynthetic membranes) a fraction of loosely (and tightly) bound water remains in pores of dry system [25, 28, 29]. Its presence manifests in NMR-sorption isotherm as a constant liquid signal already detected at $h=0$. For $R$. terebrata thalli zero-value of $A$ means the absence of "sealed" water portion in solid thallus pores. Having two different values of $k$-coefficient is consistent with paramagnetic ions on the lichen photobiont inner surfaces affecting the signal from tightly bound water differentially from that from loosely bound water.

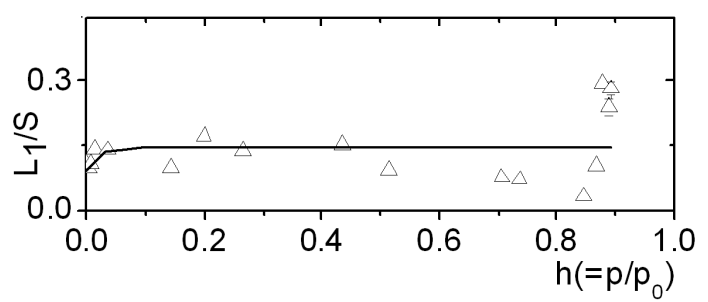

Fig. 9. The population of monolayer sorption, $L_{1} / S$, predicted by the Dent isotherm in thallus of Ramalina terebrata.

The monolayer coverage of tightly bound water sites predicted by the Dent model (Fig. 9) is given by Eq. (10) and is well fitted by the function

$$
\frac{L_{1}}{S}(h)=k \frac{\Delta M}{m_{0}} \frac{b_{1} h}{1+\left(b_{1}-b\right) h}+A,
$$

where $k$ is proportionality coefficient, and $A$ is the "sealed" water fraction. Data were fitted for $k=1.3 \pm 1.5$ and $A=0.09 \pm 0.06$. 


\section{Discussion}

Hydration courses performed from the gaseous phase, show: (i) a very tightly bound water $\left(\Delta m / m_{0}=0.007 \pm\right.$ 0.001), and, detected for higher values of target humidity: (ii) a tightly bound water $\left[\Delta m / m_{0}=0.059 \pm 0.007\right.$, hydration time $\left.t_{1}^{\mathrm{h}}=(1.2 \pm 0.2) \mathrm{h}\right]$, and finally (iii) loosely bound water pool $\left[t_{2}^{\mathrm{h}}=(14.4 \pm 2.1) \mathrm{h}\right.$, and the magnitude gradually increasing with the air humidity]. The sorption isotherm was sigmoidal in form and thus, was well described using the Dent model. The relative mass of water bound to primary binding sites was 0.046 for $R$. terebrata, which was close to sum of very tightly and tightly bound water fractions.

Both sorption isotherm models yield consistent values of the mass of water saturating primary water binding sites. Assuming that water bound to primary water binding sites forms continuous monolayer one may estimate the total inner surface of $R$. terebrata defined as water-accessible surface [8]. The obtained value equals $131.4 \mathrm{~m}^{2} / \mathrm{g}$ of dry mass. The assumption means that we get lower limit estimation.

Proton FIDs for investigated lichen reveal the presence of immobilized water fraction, $L_{1}$ and mobile water pool, $L_{2}$, which is assigned to a tightly bound water (ii), and a loosely bound water (iii) fractions. Sorption isotherm fitted to NMR data showed the absence of "sealed" water fraction trapped in pores of Ramalina terbrata and two different $k$-coefficients for a tightly $L_{1}$ and loosely $L_{2}$ bound water.

Sorption isotherm fitted to NMR data with different proportionality coefficients for tightly bound water fraction, $k_{1}$, and the one for loosely bound water fraction, $k_{2}$, shows that the signal coming from tightly bound water pool is more affected by the paramagnetic ions present in solid matrix of thallus, mostly in photosynthetic membrane of photobiont cells.

\section{References}

[1] O.L. Lange, Flora Abt B 156, 500 (1966).

[2] D.W. Larson, Can. J. Botany 56, 2119 (1978).

[3] L. Kappen, Arctic 46, 297 (1993).

[4] L. Kappen, Polar Biol. 1, 249 (1983).

[5] T.H. Nash, III, A. Reiner, B. Demmig-Adams, E. Kilian, W.M. Kaiser, O.L. Lange, New Phytol. 116, 269 (1990).

[6] L. Kappen, M. Sommerkorn, B. Schroeter, Lichenologist 7, 531 (1995).

[7] R. Del-Prado, L.G. Sancho, Flora 195, 51 (2000).

[8] H. Harańczyk, On Water in Extremely Dry Biological Systems, Wydawnictwa Uniwersytetu Jagiellońskiego, Kraków 2003.

[9] L.T. Kieft, Appl. Environ. Microbiol. 54, 1678 (1988).
[10] L.T. Kieft, V. Ahmadjian, Lichenologist 21, 355 (1989).

[11] T.L. Kieft, T. Ruscetti, T, J. Bacteriol. 172, 3519 (1990).

[12] B. Schroeter, CH. Scheidegger, New Phytol. 131, 273 (1995).

[13] D.O. Øvstedal, R.I. Lewis Smith, Lichens of Antarctica and South Georgia, Cambridge University Press, Cambridge 2001.

[14] M. Olech, Lichens of King George Island Antarctica, The Institute of Botany of the Jagiellonian University, Kraków 2004.

[15] D.F. Gaff, Oecologia (Berl.) 31, 95 (1977).

[16] W. Węglarz, H. Harańczyk, J. Phys. D, Appl. Phys. 33, 1909 (2000).

[17] R.W. Dent, Text. Res. J. 47, 145 (1977).

[18] S. Brunauer, P.H. Emmett, E. Teller, J. Am. Chem. Soc. 60, 309 (1938).

[19] H. Harańczyk, M. Bacior, M.A. Olech, Antarct. Sci. 20, 527 (2008).

[20] A. Abragam, The Principles of Nuclear Magnetism, Clarendon Press, Oxford 1961.

[21] W. Derbyshire, M. van den Bosch, D. van Dusschoten, W. MacNaughtan, I.A. Farhat, M.A. Hemminga, J.R. Mitchell, J. Magn. Res. 168, 278 (2004).

[22] H. Harańczyk, K. Strzałka, G. Jasiński, K. Mosna-Bojarska, Coll. Surf. A 115, 47 (1996).

[23] H. Harańczyk, S. Gaździński, M.A. Olech, New Phytol. 138, 191 (1998).

[24] H. Harańczyk, S. Gaździński, M.A. Olech, "Freezing protection mechanism in Cladonia mitis as observed by proton magnetic relaxation." New Aspects in Cryptogamic Research, Contribution in Honour of Ludger Kappen. Bibliotheca Lichenology 75, 265 (2000).

[25] H. Harańczyk, A. Leja, K. Strzałka, Acta Phys. Pol. A 109, 389 (2006).

[26] H. Harańczyk, A. Pietrzyk, A. Leja, M.A. Olech, Acta Phys. Pol. A 109, 411 (2006).

[27] H. Harańczyk, M. Bacior, P. Jastrzębska, M.A. Olech, Acta Phys. Pol. A 115, 516 (2009).

[28] H. Harańczyk, M. Bacior, J. Jamróz, M. Jemioła-Rzemińska, K. Strzałka, Acta Phys. Pol. A 115, 521 (2009).

[29] H. Harańczyk, A. Leja, M. Jemioła-Rzemińska, K. Strzałka, Acta Phys. Pol. A 115, 526 (2009).

[30] H. Harańczyk, J. Czak, P. Nowak, J. Nizioł, Acta Phys. Pol. A 117, 257 (2010).

[31] A. Timur, J. Petroleum Technol. 21, 775 (1969).

[32] H. Harańczyk, W.P. Węglarz, S. Sojka, Holzforschung 53, 299 (1999).

[33] M.M. Pintar, Magn. Reson. Imaging 9, 753 (1991).

[34] N. Hamada, K. Okazaki, M. Shinozaki, Bryologist 97, 176 (1994). 